\title{
Live Human Detection Robot in Earthquake Conditions
}

\author{
R. Kabilan ${ }^{\mathrm{a}, 1}$, K. Lakshmi Narayanan ${ }^{\text {a }}$, M. Venkatesh ${ }^{\mathrm{a}}$, V. Vikram Bhaskaran ${ }^{\mathrm{a}}$, \\ G. K. Viswanathan ${ }^{\text {a }}$, S. G. Yogesh Rajan ${ }^{\text {a }}$ \\ ${ }^{a}$ Dept of ECE, Francis Xavier Engineering College, Tamil Nadu, 627003, India
}

\begin{abstract}
This report outlines a human searching device that takes the form of a robotic car and serves as a backup mechanism for saving lives in the event of a disaster. The temperature sensor, in general, detects the thermal image of the human body, and there has been extensive research into human searching with the gas and humidity sensor. In the intelligent robot device's study, achieving accurate and reliable human detection and tracking is a difficult challenge. The architecture of human detection and tracking mechanisms over non-overlapping field of views is examined in this paper. To compensate for their respective flaws, a search method is proposed. The proposed method's rate and accuracy of human detection was tested in an experimental setting. We may guide the robot's movement by commanding it to move left, right, forward, or backward. We plan to equip the robot with sensors that will enable us to track and detect humans behind the wall.
\end{abstract}

Keywords. Hc-05 Bluetooth Module, Node MCU, L298D Motor drive module, Arduino UNO, De motor

\section{Introduction}

Earthquakes occur in many parts of the world, causing human injury and death [1]. Almost every year in the last decade, an earthquake of magnitude 8 or greater has struck. Around 550000 people have been reported missing or killed as a result of earthquakes during this time span [2]. As a result, demand for rescue robots is growing. Rescue robotics is a relatively recent research area, with no significant advancements until the 1990s. Rescue robots are still not commonly used in real-world search and rescue scenarios, but the field is rapidly evolving [3-7]. Finding and identifying humans under debris can be a long and difficult task, and time is critical for effective rescue operations. As just a result, fast and effective search and recovery aids are critical. There are numerous techniques for conducting urban search and rescue operations today.

\footnotetext{
${ }^{1}$ R. Kabilan, Assistant Professor, Dept of ECE, Francis Xavier Engineering College, Tamil Nadu, 627003, India; E-mail:rkabilan13@gmail.com.
} 


\section{Existing System}

We solved these concerns through equipping the UAV with just a camera for image data and a microphone for audio data, enabling us to identify people more accurately. A UAV equipped with a microphone/loudspeaker hangs above a disaster site, streaming an audio demand to all those below. The microphone picks up the voice of someone who answers, indicating if anyone needs to be rescued. A microphone and a hovering UAV, but at the other hand, were incompatible. The sound from nearby UAV rotors is picked up once by the microphone, masking the person's voice. Second, when you get further away from others, their voice becomes fainter.

To solve these issues, we must recommend sound-source detection technology to isolate the human voice by mixed sound through recorded methods [8]. An array microphone is used for sound source recording to estimate about role in human voice in sound-source difference [9]. They use results to localization to guide the UAVmounted camera placed around direction towards sound source.

\section{Proposed System}

We'll equip the robot with sensors to track humans; if an object is detected, the observed readings will deviate, indicating the presence of a human body underneath. While radars have been observed to detect and monitor objects, it is not certain that the object being tracked is an individual [10]. A Arduino microcontroller, as well as a motor, make up the device.

Via the gas, temperature, and humidity sensors, the module displays the amount of emission, humidity, and temperature of both the person and the environment. This is possible thanks to the Ada fruit website, which receives data from the sensors directly from the module [11-14]. The following readings can be easily seen on cell phones or any other electronic device. The simplest way to know the temperature, humidity, and pollution level is based on the results we get from it. Figure 1 depicts the block diagram of the proposed scheme. We will integrate the robot with sensors to track humans; if any object is detected, there will be a deviation in the observed readings, indicating the presence of a human body underneath.

\section{System Modules}

\subsection{Arduino UNO}

The Arduino UNO is the robot module's main module, and it is only via the Arduino module that all circuits and connections are accessible. The module serves as a hub for command transmission and reception. The AT mega328P microcontroller is housed on an open-source microcontroller board. There is $1 \mathrm{~KB}$ of ROM, $2 \mathrm{~KB}$ data of RAM, and $32 \mathrm{~KB}$ on flash memory on the board.

\subsection{Node $M C U$}

The Arduino is linked to the node MCU, which is a strong WIFI module. It's also an IoT framework that's open source. It consists of an express-if systems system on a chip 
and hardware based upon ESP-12 module. Rather than development kits, the name "Node MCU" is commonly used to refer to the firmware.

\subsection{Bluetooth module}

The Bluetooth module in this device allows us to connect it to a mobile phone via a mobile application, as well as monitor all of the movements, including forward, backward, left, and right, with pinpoint accuracy. The Bluetooth module and the intermediate serial port protocol module are both called Hc-05. This communicates through serial communication, which makes it simple to use.

\subsection{Bluetooth module}

The Dc motor is steered in either direction using the L298D motor drive IC. It has 16 pins and can drive two DC motors with a single integrated circuit.

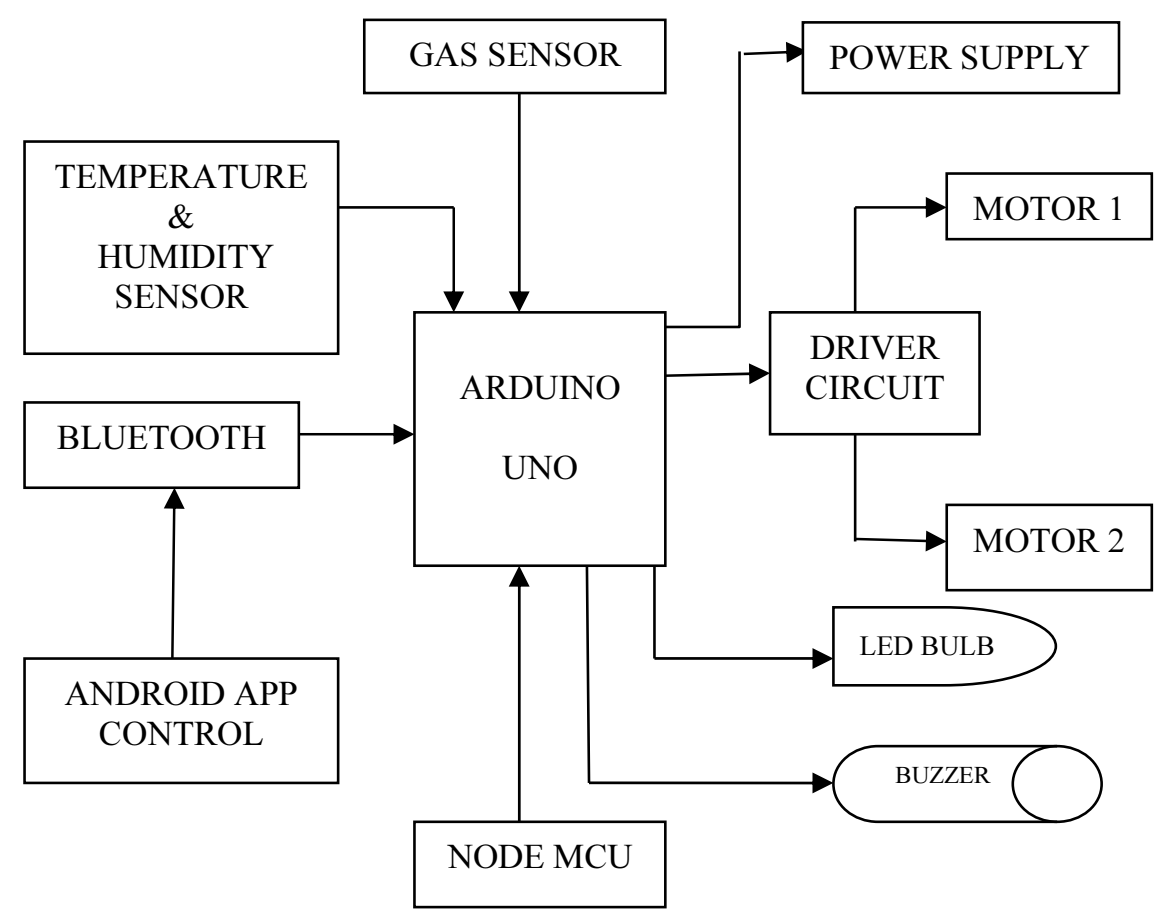

Figure 1. Block Diagram of proposed system 


\section{Hardware Description}

There are 14 digital Input, output pins for accessing data to the Arduino board, as well as 6 analogue input pins. An Arduino's GND (\#) pins serve which serves to be negative terminal for circuits or sensors attached on it. An $3.3 \mathrm{~V}$ and $5 \mathrm{~V}$ pins provide 5 and $3.3 \mathrm{~V}$ of power applied to circuit, accordingly. By vast majority of sensors required set up an operate on two voltages. A power source is required for all boards to function. The board can be driven in two ways. The first method is to connected to USB cable with device. The board could also be worked by attached with the wall on power supply towards barrel jack. Additionally, code of the ATmega328 microchip is uploaded via USB.

Both Arduino device need a power supply for the code on the following chipset, which connects to the module's respective pin. On the Arduino UNO, the digital I/O pins are numbered from 0 to 13. It is used in digital inputs, such as data acceptance. The Arduino UNO, is also called as microprocessor module, ATmega328P is an microcontroller. Board is made up of various ram, rom, and flash memory components, as well as the Arduino IDE programming software. They also provide digital pins for modifying previously written data on the feature with the file of pins in the section of each Arduino digital I/O reading and writing way.

The DC motor is driven by the L298D motor drive IC, which allows this to drive in any path whereas the robot is operated. An H-Bridge circuit in a single L293D it has couple of DC motors simultaneously. Four inputs pins were in charge of control all rotation of attached motors. The input pins has either logic 1 or logic 0 which is adjust towards the direction. Normally it is 16-pin motor drive and has IC it can power 2 sets of DC motor which can operate in any direction at same time. In both directions, the L293D is modeled as bidirectional drive current to maximum upto $600 \mathrm{~mA}$.

\section{Results}

In our proposed method, we also use a multimeter to measure output voltage, input voltage and current. LUO converter used as quick charging method. The LUO converter receives a $12 \mathrm{~V}$ AC input voltage as a single input. The output voltage must be 24 volts direct current. However, the harmonic can be reduced. When the converter is set to $10 \mathrm{amps}$, the input current will be $26.5 \mathrm{amps}$, as shown in Figure 2. The output voltage must be 24 volts direct current. Moreover, since this current is needed for fast charging, the required voltage at output will be $1.5 \mathrm{~V}$ DC with $26.5 \mathrm{~V}$.
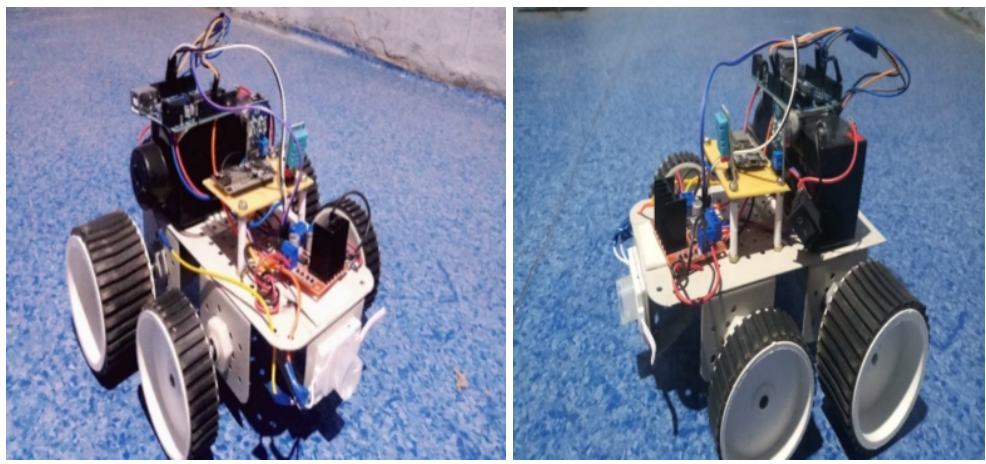


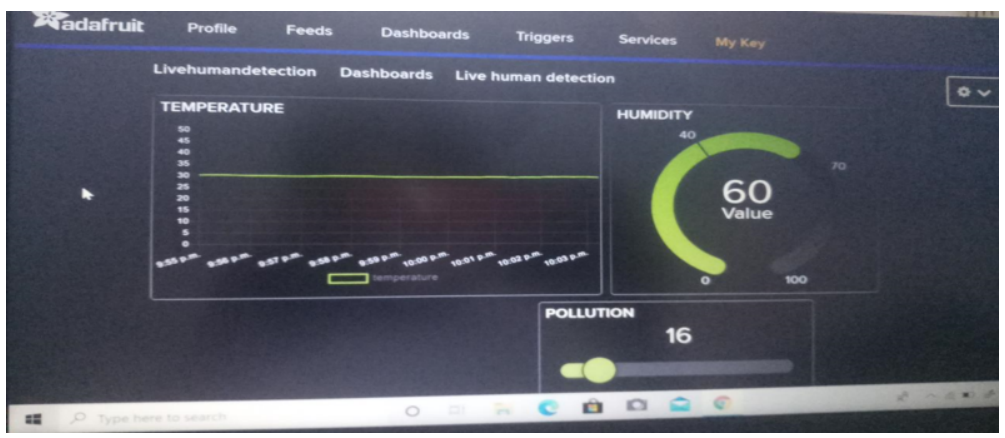

Figure 2. Shows the side view of Live Human Detection and system result

\section{Conclusion}

The proposed robotic device can able to capture of human lives on detection of earthquake along location tracking, environmental current condition monitoring and live streaming was developed with the Raspberry PI with an intermediate processor. The developed device has validated and has covered the benefits of many existing systems. The machine can also perform rescue operations in the event of landslides and avalanches. Since Internet access would be low in these locations, it is preferable to use radar communication in a real-time system rather than IOT. We created a low-cost autonomously operated rescue robot for this project. Search and rescue operations can be made more successful and efficient with the aid of this robot. After putting the module together, it was double-checked for functionality. A prototype robotic device capable of post-earthquake human live detection as location tracking, environmental current condition monitoring, and live streaming was developed with the Raspberry PI like intermediate processor. The developed device has been validated and has covered the benefits of many existing systems. The machine can also perform rescue operations in the event of landslides and avalanches. Since Internet access would be low in these locations, it is preferable to use radar communication in a real-time system rather than IOT. We created a low-cost autonomously operated rescue robot for this project. Search and rescue operations can be made more successful and efficient with the aid of this robot. After putting the module together, it was double-checked for functionality. The developed device has validated and has covered the benefits of many existing systems. The machine can also perform rescue operations in the event of landslides and avalanches. Since Internet access would be low in these locations, it is preferable to use radar communication in a real-time system rather than IOT. Many lives will be saved and the autonomous module could be used for several purposes in the real-time system if it is upgraded to the highest quality.

\section{References}

[1] Benli, E., Motai, Y., \& Rogers, J. (2019). Human Behavior-Based Target Tracking With an OmniDirectional Thermal Camera. IEEE Transactions on Cognitive and Developmental Systems, 11(1), 3650. https://doi.org/10.1109/TCDS.2017.2726356

[2] Booranawong, A., Jindapetch, N., \& Saito, H. (2019). Adaptive Filtering Methods for RSSI Signals in a Device-Free Human Detection and Tracking System. IEEE Systems Journal, 13(3), 2998-3009. https://doi.org/10.1109/JSYST.2019.2919642 
[3] Booranawong, A., Jindapetch, N., \& Saito, H. (2018). A System for Detection and Tracking of Human Movements Using RSSI Signals. IEEE Sensors Journal, 18(6), 2531-2544. https://doi.org/10.1109/JSEN.2018.2795747

[4] Chang, F.-M., Lian, F.-L., \& Chou, C.-C. (2016). Integration of Modified Inverse Observation Model and Multiple Hypothesis Tracking for Detecting and Tracking Humans. IEEE Transactions on Automation Science and Engineering, 13(1), 160-170. https://doi.org/10.1109/TASE.2015.2426712

[5] Chen, Z., Wang, Y., \& Liu, H. (2018). Unobtrusive Sensor-Based Occupancy Facing Direction Detection and Tracking Using Advanced Machine Learning Algorithms. IEEE Sensors Journal, 18(15), 6360-6368. https://doi.org/10.1109/JSEN.2018.2844252

[6] Huang, C.-H. P., Allain, B., Boyer, E., Franco, J.-S., Tombari, F., Navab, N., \& Ilic, S. (2018). Tracking-by-Detection of 3D Human Shapes: From Surfaces to Volumes. IEEE Transactions on Pattern Analysis and Machine Intelligence, 40(8), 1994-2008. https://doi.org/10.1109/TPAMI.2017.2740308

[7] Li, F., Chen, C.-H., Xu, G., \& Khoo, L.-P. (2020). Hierarchical Eye-Tracking Data Analytics for Human Fatigue Detection at a Traffic Control Center. IEEE Transactions on Human-Machine Systems, 50(5), 465-474. https://doi.org/10.1109/THMS.2020.3016088

[8] Li, H., Cui, G., Kong, L., Chen, G., Wang, M., \& Guo, S. (2019). Robust Human Targets Tracking for MIMO Through-Wall Radar via Multi-Algorithm Fusion. IEEE Journal of Selected Topics in Applied Earth Observations and Remote Sensing, 12(4), 1154-1164. https://doi.org/10.1109/JSTARS.2019.2901262

[9] Li, H., Cui, G., Kong, L., Guo, S., \& Wang, M. (2020). Scale-Adaptive Human Target Tracking for Through-Wall Imaging Radar. IEEE Geoscience and Remote Sensing Letters, 17(8), 1348-1352. https://doi.org/10.1109/LGRS.2019.2948629

[10] Paral, P., Chatterjee, A., \& Rakshit, A. (2019). Vision Sensor-Based Shoe Detection for Human Tracking in a Human-Robot Coexisting Environment: A Photometric Invariant Approach Using DBSCAN Algorithm. IEEE Sensors Journal, 19(12), 4549-4559. https://doi.org/10.1109/JSEN.2019.2897989

[11] Will, C., Vaishnav, P., Chakraborty, A., \& Santra, A. (2019). Human Target Detection, Tracking, and Classification Using 24-GHz FMCW Radar. IEEE Sensors Journal, 19(17), 7283-7299. https://doi.org/10.1109/JSEN.2019.2914365

[12] Yang, B., \& Zhang, M. (2017). Credit-Based Multiple Human Location for Passive Binary Pyroelectric Infrared Sensor Tracking System: Free From Region Partition and Classifier. IEEE Sensors Journal, 17(1), 37-45. https://doi.org/10.1109/JSEN.2016.2623325

[13] Yun, J., \& Woo, J. (2020). A Comparative Analysis of Deep Learning and Machine Learning on Detecting Movement Directions Using PIR Sensors. IEEE Internet of Things Journal, 7(4), 2855-2868. https://doi.org/10.1109/JIOT.2019.2963326

[14] Zhang, Y. (2020). Detection and Tracking of Human Motion Targets in Video Images Based on Camshift Algorithms. IEEE Sensors Journal, 20(20), 11887-11893. https://doi.org/10.1109/JSEN.2019.2956051 